\title{
Lower semicontinuity of approximate solution mappings for parametric generalized vector equilibrium problems
}

\author{
Rabian Wangkeeree ${ }^{1 *}$, Panatda Boonman ${ }^{1}$ and Pakkapon Preechasilp ${ }^{2}$
}

\section{"Correspondence:}

rabianw@nu.ac.th

'Department of Mathematics,

Faculty of Science, Naresuan

University, Phitsanulok, 65000,

Thailand

Full list of author information is

available at the end of the article

\begin{abstract}
In this paper, we obtain sufficient conditions for the lower semicontinuity of an approximate solution mapping for a parametric generalized vector equilibrium problem involving set-valued mappings. By using a scalarization method, we obtain the lower semicontinuity of an approximate solution mapping for such a problem without the assumptions of monotonicity and compactness.
\end{abstract}

Keywords: lower semicontinuity; approximate solution mapping; parametric generalized vector equilibrium problems; scalarization method

\section{Introduction}

The vector equilibrium problem is a unified model of several problems, for example, the vector optimization problem, the vector variational inequality problem, the vector complementarity problem and the vector saddle point problem. In the literature, existence results for various types of vector equilibrium problems have been investigated intensively, e.g., see [1-4] and the references therein. The stability analysis of the solution mappings for VEP is an important topic in vector equilibrium theory. Recently, the semicontinuity, especially the lower semicontinuity, of solution mappings to parametric vector equilibrium problems has been studied in the literature, see [5-16]. In the mentioned results, the lower semicontinuity of solution mappings to parametric generalized strong vector equilibrium problems is established under the assumptions of monotonicity and compactness. Very recently, Han and Gong [17] studied the lower semicontinuity of solution mappings to parametric generalized strong vector equilibrium problems without the assumptions of monotonicity and compactness.

On the other hand, exact solutions of the problems may not exist in many practical problems because the data of the problems are not sufficiently 'regular.' Moreover, these mathematical models are solved usually by numerical methods which produce approximations to the exact solutions. So it is impossible to obtain an exact solution of many practical problems. Naturally, investigating approximate solutions of parametric equilibrium problems is of interest in both practical applications and computations. Anh and Khanh [18] considered two kinds of approximate solution mappings to parametric generalized vector quasiequilibrium problems and established the sufficient conditions for their Hausdorff semicontinuity (or Berge semicontinuity). Among many approaches for dealing with the

○2014 Wangkeeree et al.; licensee Springer. This is an Open Access article distributed under the terms of the Creative Commons Attribution License (http://creativecommons.org/licenses/by/2.0), which permits unrestricted use, distribution, and reproduction in any medium, provided the original work is properly cited. 
lower semicontinuity and continuity of solution mappings for parametric vector variational inequalities and parametric vector equilibrium problems, the scalarization method is of considerable interest. By using a scalarization method, Li and Li [19] discussed the Berge lower semicontinuity and Berge continuity of an approximate solution mapping for a parametric vector equilibrium problem.

Motivated by the work reported in [17-19], in this paper we aim to establish efficient conditions for the lower semicontinuity of an approximate solution mapping for a parametric generalized vector equilibrium problem involving set-valued mappings. By using a scalarization method, we obtain the lower semicontinuity of an approximate solution mapping for such a problem without the assumptions of monotonicity and compactness.

\section{Preliminaries}

Throughout this paper, let $X$ and $Y$ be real Hausdorff topological vector spaces, and let $Z$ be a real topological space. We also assume that $C$ is a pointed closed convex cone in $Y$ with its interior int $C \neq \emptyset$. Let $Y^{*}$ be the topological dual space of $Y$. Let $C^{*}:=\left\{\xi \in Y^{*}:\langle\xi, y\rangle \geq\right.$ $0, \forall y \in C\}$ be the dual cone of $C$, where $\langle\xi, y\rangle$ denotes the value of $\xi$ at $y$. Since int $C \neq \emptyset$, the dual cone $C^{*}$ of $C$ has a weak* compact base. Let $e \in \operatorname{int} C$. Then $B_{e}^{*}:=\left\{\xi \in C^{*}:\langle\xi, e\rangle=1\right\}$ is a weak* compact base of $C^{*}$.

Suppose that $K$ is a nonempty subset of $X$ and $F: K \times K \rightarrow 2^{Y} \backslash\{\emptyset\}$ is a set-valued mapping. We consider the following generalized vector equilibrium problem (GVEP) of finding $x_{0} \in K$ such that

$$
F\left(x_{0}, y\right) \subset Y \backslash-\operatorname{int} C, \quad \forall y \in K \text {. }
$$

When the set $K$ and the mapping $F$ are perturbed by a parameter $\mu$ which varies over a set $M$ of $Z$, we consider the following parametric generalized vector equilibrium problem (PGVEP) of finding $x_{0} \in K(\mu)$ such that

$$
F\left(x_{0}, y, \mu\right) \subset Y \backslash-\operatorname{int} C, \quad \forall y \in K(\mu),
$$

where $K: M \rightarrow 2^{X} \backslash\{\emptyset\}$ is a set-valued mapping, $F: B \times B \times M \subset X \times X \times Z \rightarrow 2^{Y} \backslash\{\emptyset\}$ is a set-valued mapping with $K(M)=\bigcup_{\mu \in M} K(\mu) \subset B$. For each $\varepsilon>0$ and $\mu \in M$, the approximate solution set of (PGVEP) is defined by

$$
\widetilde{S}(\varepsilon, \mu):=\{x \in K(\mu): F(x, y, \mu)+\varepsilon e \subset Y \backslash-\operatorname{int} C, \forall y \in K(\mu)\},
$$

where $e \in \operatorname{int} C$. For each $\xi \in B_{e}^{*}$ and $(\varepsilon, \mu) \in \mathbb{R}^{+} \times M$, by $\widetilde{S}_{\xi}(\varepsilon, \mu)$ we denote the $\xi$-approximate solution set of (PGVEP), i.e.,

$$
\widetilde{S}_{\xi}(\varepsilon, \mu):=\left\{x \in K(\mu): \inf _{z \in F(x, y, \mu)} \xi(z)+\varepsilon \geq 0, \forall y \in K(\mu)\right\} .
$$

Definition 2.1 Let $D$ be a nonempty convex subset of $X$. A set-valued mapping $G: X \rightarrow$ $2^{Y}$ is said to be:

(i) C-convex on $D$ if, for any $x_{1}, x_{2} \in D$ and for any $t \in[0,1]$, we have

$$
t G\left(x_{1}\right)+(1-t) G\left(x_{2}\right) \subseteq G\left(t x_{1}+(1-t) x_{2}\right)+C .
$$


(ii) $C$-concave on $D$ if, for any $x_{1}, x_{2} \in D$ and for any $t \in[0,1]$, we have

$$
G\left(t x_{1}+(1-t) x_{2}\right) \subseteq t G\left(x_{1}\right)+(1-t) G\left(x_{2}\right)+C
$$

Definition 2.2 [17] Let $M$ and $M_{1}$ be topological vector spaces. Let $D$ be a nonempty subset of $M$. A set-valued mapping $G: M \rightarrow 2^{M_{1}}$ is said to be uniformly continuous on $D$ if, for any neighborhood $V$ of $0 \in M_{1}$, there exists a neighborhood $U_{0}$ of $0 \in M$ such that $G\left(x_{1}\right) \subseteq G\left(x_{2}\right)+V$ for any $x_{1}, x_{2} \in D$ with $x_{1}-x_{2} \in U_{0}$.

Definition 2.3 [20] Let $M$ and $M_{1}$ be topological vector spaces. A set-valued mapping $G: M \rightarrow 2^{M_{1}}$ is said to be:

(i) Hausdorff upper semicontinuous (H-u.s.c.) at $u_{0} \in M$ if, for any neighborhood $V$ of $0 \in M_{1}$, there exists a neighborhood $U\left(u_{0}\right)$ of $u_{0}$ such that

$$
G(u) \subseteq G\left(u_{0}\right)+V \quad \text { for every } u \in U\left(u_{0}\right)
$$

(ii) Lower semicontinuous (l.s.c.) at $u_{0} \in M$ if, for any $x \in G\left(u_{0}\right)$ and any neighborhood $V$ of $x$, there exists a neighborhood $U\left(u_{0}\right)$ of $u_{0}$ such that

$$
G(u) \cap V \neq \emptyset \quad \text { for every } u \in U\left(u_{0}\right) \text {. }
$$

The following lemma plays an important role in the proof of the lower semicontinuity of the solution mapping $\widetilde{S}(\cdot, \cdot)$.

Lemma 2.4 [21, Theorem 2] The union $\Gamma=\bigcup_{i \in I} \Gamma_{i}$ of a family ofl.s.c. set-valued mappings $\Gamma_{i}$ from a topological space $X$ into a topological space $Y$ is also an l.s.c. set-valued mapping from $X$ into $Y$, where $I$ is an index set.

\section{Lower semicontinuity of the approximate solution mapping for (PGVEP)}

In this section, we establish the lower semicontinuity of the approximate solution mapping for (PGVEP) at the considered point $\left(\varepsilon_{0}, \mu_{0}\right) \in \mathbb{R}^{+} \times M$ with $\varepsilon_{0}>0$.

Firstly, using the same argument as in the proof given in [22, Lemma 3.1], we can prove the following useful result.

Lemma 3.1 For each $\varepsilon>0, \mu \in M$, iffor each $x \in K(\mu), F(x, K(\mu), \mu)+C$ is a convex set, then

$$
\widetilde{S}(\varepsilon, \mu)=\bigcup_{\xi \in C^{*} \backslash\{0\}} \widetilde{S}_{\xi}(\varepsilon, \mu)=\bigcup_{\xi \in B_{e}^{*}} \widetilde{S}_{\xi}(\varepsilon, \mu) .
$$

Proof For any $x \in \bigcup_{\xi \in C^{*} \backslash\{0\}} \widetilde{S}_{\xi}(\varepsilon, \mu)$, there exists $\xi^{\prime} \in C^{*} \backslash\{0\}$ such that $x \in \widetilde{S}_{\xi^{\prime}}(\varepsilon, \mu)$. Thus, we can obtain that $x \in K(\mu)$ and $\inf _{z \in F(x, y, \mu)} \xi^{\prime}(z)+\varepsilon \geq 0, \forall y \in K(\mu)$. Then, for each $y \in$ $K(\mu)$ and $z \in F(x, y, \mu), \xi^{\prime}(z)+\varepsilon \geq 0$, which arrives at $z \notin-\operatorname{int} C$. It then follows that, for each $z \in F(x, y, \mu)$,

$$
F(x, y, \mu)+\varepsilon e \subseteq Y \backslash-\operatorname{int} C, \quad \forall y \in K(\mu),
$$


which gives that $x \in \widetilde{S}(\varepsilon, \mu)$. Hence, $\bigcup_{\xi \in C^{*} \backslash\{0\}} \widetilde{S}_{\xi}(\varepsilon, \mu) \subseteq \widetilde{S}(\varepsilon, \mu)$. Conversely, let $x \in \widetilde{S}(\varepsilon, \mu)$ be arbitrary. Then $x \in K(\mu)$ and $F(x, y, \mu)+\varepsilon e \subseteq Y \backslash-\operatorname{int} C, \forall y \in K(\mu)$. Thus, we have

$$
F(x, K(\mu), \mu) \cap(-\operatorname{int} C)=\emptyset,
$$

and hence

$$
(F(x, K(\mu), \mu)+C) \cap(-\operatorname{int} C)=\emptyset .
$$

Because $F(x, K(\mu), \mu)+C$ is a convex set, by the well-known Edidelheit separation theorem (see [23], Theorem 3.16), there exist a continuous linear functional $\xi \in Y^{*} \backslash\{0\}$ and a real number $\gamma$ such that

$$
\xi(\hat{c})<\gamma \leq \xi(z+c)
$$

for all $z \in F(x, K(\mu), \mu), c \in C$ and $\hat{c} \in-\operatorname{int} C$. Since $C$ is a cone, we have $\xi(\hat{c}) \leq 0$ for all $\hat{c} \in-\operatorname{int} C$. Thus, $\xi(\hat{c}) \geq 0$ for all $\hat{c} \in C$, that is, $\xi \in C^{*}$. Moreover, it follows from $c \in C$, $\hat{c} \in-\operatorname{int} C$ and the continuity of $\xi$ that $\xi(z)+\varepsilon \geq 0$ for all $z \in F(x, K(\mu), \mu)$. Thus, for all $y \in K(\mu)$, we have $\inf _{z \in F(x, y, \mu)} \xi(z)+\varepsilon \geq 0$, i.e., $x \in \widetilde{S}_{\xi}(\varepsilon, \mu) \subseteq \bigcup_{\xi \in C^{*} \backslash\{0\}} \widetilde{S}_{\xi}(\varepsilon, \mu)$.

Theorem 3.2 We assume that for any given $\xi \in B_{e}^{*}$, there exists $\delta>0$ such that the $\xi$-approximate solution set $\widetilde{S}_{\xi}(\cdot, \cdot)$ exists in $\left[\varepsilon_{0}, \delta\right) \times N\left(\mu_{0}\right)$, where $N\left(\mu_{0}\right)$ is a neighborhood of $\mu_{0}$. Assume further that the following conditions are satisfied:

(i) $K\left(\mu_{0}\right)$ is nonempty convex;

(ii) $K$ is $H$-u.s.c. at $\mu_{0}$ and l.s.c. at $\mu_{0}$;

(iii) for any $y \in K\left(\mu_{0}\right), F\left(\cdot, y, \mu_{0}\right)$ is $C$-concave on $K\left(\mu_{0}\right)$;

(iv) $F(\cdot, \cdot, \cdot)$ is uniformly continuous on $K(M) \times K(M) \times N\left(\mu_{0}\right)$.

Then the $\xi$-approximate solution mapping $\widetilde{S}_{\xi}:\left[\varepsilon_{0}, \delta\right) \times N\left(\mu_{0}\right) \rightarrow 2^{X}$ is l.s.c. at $\left(\varepsilon_{0}, \mu_{0}\right)$.

Proof Suppose to the contrary that $\widetilde{S}_{\xi}(\cdot, \cdot)$ is not l.s.c. at $\left(\varepsilon_{0}, \mu_{0}\right)$, then there exist $x_{0} \in$ $\widetilde{S}_{\xi}\left(\varepsilon_{0}, \mu_{0}\right)$ and a neighborhood $W_{0}$ of $0_{X} \in X$. For any neighborhoods $J\left(\varepsilon_{0}\right)$ and $U\left(\mu_{0}\right)$ of $\varepsilon_{0}$ and $\mu_{0}$, respectively, there exist $\varepsilon^{\prime} \in J\left(\varepsilon_{0}\right) \cap\left[\varepsilon_{0}, \delta\right)$ and $\mu^{\prime} \in U\left(\mu_{0}\right)$ such that $\left(x_{0}+W_{0}\right) \cap$ $\widetilde{S}_{\xi}\left(\varepsilon^{\prime}, \mu^{\prime}\right)=\emptyset$. In particular, there exist sequences $\left\{\varepsilon_{n}\right\} \downarrow \varepsilon_{0}$ and $\left\{\mu_{n}\right\} \rightarrow \mu_{0}$ such that

$$
\left(x_{0}+W_{0}\right) \cap \widetilde{S}_{\xi}\left(\varepsilon_{n}, \mu_{n}\right)=\emptyset, \quad \forall n \in \mathbb{N} .
$$

For the above $W_{0}$, there exists a neighborhood $W_{1}$ of $0_{X} \in X$ such that

$$
W_{1}+W_{1} \subseteq W_{0}
$$

We define a $\xi$-set-valued mapping $H_{\xi}:[0, \delta) \rightarrow 2^{X}$ by

$$
H_{\xi}(\varepsilon)=\left\{x \in K\left(\mu_{0}\right): \inf _{z \in F\left(x, y, \mu_{0}\right)} \xi(z)+\varepsilon+\varepsilon_{0} \geq 0, \forall y \in K\left(\mu_{0}\right)\right\}, \quad \varepsilon \in[0, \delta) .
$$

Notice that $H_{\xi}(0)=\widetilde{S}_{\xi}\left(\varepsilon_{0}, \mu_{0}\right) \neq \emptyset$. Next, we claim that $H_{\xi}$ is l.s.c. at 0 . Suppose to the contrary that $H_{\xi}$ is not l.s.c. at 0 , then there exist $\bar{x} \in H_{\xi}(0)$ and a neighborhood $O_{0}$ of 
$0_{X} \in X$. For any neighborhood $U$ of 0 , there exists $\varepsilon \in U$ such that $\left(\bar{x}+O_{0}\right) \cap H_{\xi}(\varepsilon)=\emptyset$. In particular, there exists a nonnegative sequence $\left\{\varepsilon_{n}^{\prime}\right\} \downarrow 0$ such that

$$
\left(\bar{x}+O_{0}\right) \cap H_{\xi}\left(\varepsilon_{n}^{\prime}\right)=\emptyset, \quad \forall n \in \mathbb{N} .
$$

Since $H_{\xi}(0) \neq \emptyset$, we choose $x^{*} \in H_{\xi}(0)$. Since $\varepsilon_{n}^{\prime} \rightarrow 0$, there exists $\varepsilon_{n_{0}}^{\prime}$ such that

$$
\frac{\varepsilon_{0}}{\varepsilon_{0}+\varepsilon_{n_{0}}^{\prime}} \bar{x}+\frac{\varepsilon_{n_{0}}^{\prime}}{\varepsilon_{0}+\varepsilon_{n_{0}}^{\prime}} x^{*}=\bar{x}+\frac{\varepsilon_{n_{0}}^{\prime}}{\varepsilon_{0}+\varepsilon_{n_{0}}^{\prime}}\left(x^{*}-\bar{x}\right) \in \bar{x}+O_{0} .
$$

We claim that $\frac{\varepsilon_{0}}{\varepsilon_{0}+\varepsilon_{n_{0}}^{\prime}} \bar{x}+\frac{\varepsilon_{n_{0}}^{\prime}}{\varepsilon_{0}+\varepsilon_{n_{0}}^{\prime}} x^{*} \in H_{\xi}\left(\varepsilon_{n_{0}}^{\prime}\right)$. In fact, since $\bar{x} \in H_{\xi}(0)$ and $x^{*} \in H_{\xi}(0)$, for any $y \in K\left(\mu_{0}\right)$, we have $\inf _{t \in F\left(\bar{x}, y, \mu_{0}\right)} \xi(t)+\varepsilon_{0} \geq 0$ and $\inf _{k \in F\left(x^{*}, y, \mu_{0}\right)} \xi(k)+\varepsilon_{0} \geq 0$. Then, for any $u \in F\left(\bar{x}, y, \mu_{0}\right)$,

$$
\frac{\varepsilon_{0}}{\varepsilon_{0}+\varepsilon_{n_{0}}^{\prime}} \xi(u)+\frac{\varepsilon_{0}}{\varepsilon_{0}+\varepsilon_{n_{0}}^{\prime}} \varepsilon_{0} \geq 0
$$

and for any $v \in F\left(x^{*}, y, \mu_{0}\right)$,

$$
\frac{\varepsilon_{n_{0}}^{\prime}}{\varepsilon_{0}+\varepsilon_{n_{0}}^{\prime}} \xi(v)+\frac{\varepsilon_{n_{0}}^{\prime}}{\varepsilon_{0}+\varepsilon_{n_{0}}^{\prime}} \varepsilon_{0} \geq 0
$$

By the $C$-concavity of $F\left(\cdot, y, \mu_{0}\right)$, we have that

$$
F\left(\frac{\varepsilon_{0}}{\varepsilon_{0}+\varepsilon_{n_{0}}^{\prime}} \bar{x}+\frac{\varepsilon_{n_{0}}^{\prime}}{\varepsilon_{0}+\varepsilon_{n_{0}}^{\prime}} x^{*}, y, \mu_{0}\right) \subseteq \frac{\varepsilon_{0}}{\varepsilon_{0}+\varepsilon_{n_{0}}^{\prime}} F\left(\bar{x}, y, \mu_{0}\right)+\frac{\varepsilon_{n_{0}}^{\prime}}{\varepsilon_{0}+\varepsilon_{n_{0}}^{\prime}} F\left(x^{*}, y, \mu_{0}\right)+C .
$$

It follows that, for any $w \in F\left(\frac{\varepsilon_{0}}{\varepsilon_{0}+\varepsilon_{n_{0}}^{\prime}} \bar{x}+\frac{\varepsilon_{n_{0}}^{\prime}}{\varepsilon_{0}+\varepsilon_{n_{0}}^{\prime}} x^{*}, y, \mu_{0}\right)$, there exist $\bar{z} \in F\left(\bar{x}, y, \mu_{0}\right), z^{*} \in$ $F\left(x^{*}, y, \mu_{0}\right)$ and $c^{\prime} \in C$ such that $w=\frac{\varepsilon_{0}}{\varepsilon_{0}+\varepsilon_{n_{0}}^{\prime}} \bar{z}+\frac{\varepsilon_{n_{0}}^{\prime}}{\varepsilon_{0}+\varepsilon_{n_{0}}^{\prime}} z^{*}+c^{\prime}$. It follows from the linearity of $\xi$ that $\xi(w)-\frac{\varepsilon_{0}}{\varepsilon_{0}+\varepsilon_{n_{0}}^{\prime}} \xi(\bar{z})-\frac{\varepsilon_{n_{0}}^{\prime}}{\varepsilon_{0}+\varepsilon_{n_{0}}^{\prime}} \xi\left(z^{*}\right)=\xi\left(c^{\prime}\right) \geq 0$, which gives that $\xi(w) \geq \frac{\varepsilon_{0}}{\varepsilon_{0}+\varepsilon_{n_{0}}^{\prime}} \xi(\bar{z})+$ $\frac{\varepsilon_{n_{0}}^{\prime}}{\varepsilon_{0}+\varepsilon_{n_{0}}^{\prime}} \xi\left(z^{*}\right)$. For all $w \in F\left(\frac{\varepsilon_{0}}{\varepsilon_{0}+\varepsilon_{n_{0}}^{\prime}} \bar{x}+\frac{\varepsilon_{n_{0}}^{\prime}}{\varepsilon_{0}+\varepsilon_{n_{0}}^{\prime}} x^{*}, y, \mu_{0}\right)$, by (3.5) and (3.6), we have

$$
\xi(w) \geq-\frac{\varepsilon_{0}}{\varepsilon_{0}+\varepsilon_{n_{0}}^{\prime}} \varepsilon_{0}-\frac{\varepsilon_{n_{0}}^{\prime}}{\varepsilon_{0}+\varepsilon_{n_{0}}^{\prime}} \varepsilon_{0}=-\frac{\varepsilon_{0}}{\varepsilon_{0}+\varepsilon_{n_{0}}^{\prime}}\left(\varepsilon_{n_{0}}^{\prime}+\varepsilon_{0}\right) \geq-\left(\varepsilon_{n_{0}}^{\prime}+\varepsilon_{0}\right) .
$$

This implies that $\inf _{z \in F\left(\frac{\varepsilon_{0}}{\varepsilon_{0}+\varepsilon_{n_{0}}^{\prime}} \bar{x}+\frac{\varepsilon_{n_{0}}^{\prime}}{\varepsilon_{0}+\varepsilon_{n_{0}}^{\prime}} x^{*}, y, \mu_{0}\right)} \xi(z)+\varepsilon_{n_{0}}^{\prime}+\varepsilon_{0} \geq 0$, that is, $\frac{\varepsilon_{0}}{\varepsilon_{0}+\varepsilon_{n_{0}}^{\prime}} \bar{x}+\frac{\varepsilon_{n_{0}}^{\prime}}{\varepsilon_{0}+\varepsilon_{n_{0}}^{\prime}} x^{*} \in$ $H_{\xi}\left(\varepsilon_{n_{0}}^{\prime}\right)$. By (3.4), we get that $\frac{\varepsilon_{0}}{\varepsilon_{0}+\varepsilon_{n_{0}}^{\prime}} \bar{x}+\frac{\varepsilon_{n_{0}}^{\prime}}{\varepsilon_{0}+\varepsilon_{n_{0}}^{\prime}} x^{*} \in\left(\bar{x}+O_{0}\right) \cap H_{\xi}\left(\varepsilon_{n_{0}}^{\prime}\right)$, which contradicts (3.3). Therefore, $H_{\xi}$ is l.s.c. at 0 . Since $H_{\xi}$ is l.s.c. at 0 , for above $x_{0} \in \widetilde{S}_{\xi}\left(\varepsilon_{0}, \mu_{0}\right)=$ $H_{\xi}(0)$ and for above $W_{1}$, there exists a balanced neighborhood $V_{0}$ of 0 such that $\left(x_{0}+\right.$ $\left.W_{1}\right) \cap H_{\xi}(\varepsilon) \neq \emptyset, \forall \varepsilon \in V_{0}$. In particular, from $\left\{\varepsilon_{n}\right\} \downarrow \varepsilon_{0}$, there exits $N_{0} \in \mathbb{N}$ such that $\left(x_{0}+W_{1}\right) \cap H_{\xi}\left(\varepsilon_{N_{0}}-\varepsilon_{0}\right) \neq \emptyset$. Let $x^{\prime} \in\left(x_{0}+W_{1}\right) \cap H_{\xi}\left(\varepsilon_{N_{0}}-\varepsilon_{0}\right)$.

For any $\bar{\varepsilon}>0$, since $e \in \operatorname{int} C$, there exists $\delta_{0}>0$ such that

$$
\delta_{0} B_{Y}+\bar{\varepsilon} e \subseteq C
$$


Since $F(\cdot, \cdot, \cdot)$ is uniformly continuous on $K(M) \times K(M) \times N\left(\mu_{0}\right)$, for above $\delta_{0} B_{Y}$, there exists a neighborhood $V_{1}$ of $0 \in B$, a neighborhood $U_{1}$ of $0 \in B$ and a neighborhood $N_{1}$ of $0 \in M$, for any $\left(x_{1}, y_{1}, \mu_{1}\right),\left(x_{2}, y_{2}, \mu_{2}\right) \in K(M) \times K(M) \times N\left(\mu_{0}\right)$ with $x_{1}-x_{2} \in V_{1}, y_{1}-y_{2} \in U_{1}$ and $\mu_{1}-\mu_{2} \in N_{1}$, we have

$$
F\left(x_{1}, y_{1}, \mu_{1}\right) \subseteq \delta_{0} B_{Y}+F\left(x_{2}, y_{2}, \mu_{2}\right)
$$

Since $K$ is H-u.s.c. at $\mu_{0}$, for above $U_{1}$, there exists a neighborhood $U_{1}\left(\mu_{0}\right)$ of $\mu_{0}$ such that

$$
K(\mu) \subseteq K\left(\mu_{0}\right)+U_{1}, \quad \forall \mu \in U_{1}\left(\mu_{0}\right) .
$$

We see that $x^{\prime} \in K\left(\mu_{0}\right)$. Since $K$ is l.s.c. at $\mu_{0}$, for $V_{1} \cap W_{1}$, there exists a neighborhood $U_{2}\left(\mu_{0}\right)$ of $\mu_{0}$ such that

$$
\left(x^{\prime}+V_{1} \cap W_{1}\right) \cap K(\mu) \neq \emptyset, \quad \forall \mu \in U_{2}\left(\mu_{0}\right) .
$$

It follows from $\mu_{n} \rightarrow \mu_{0}$ that there exists a positive integer $N_{0}^{\prime} \geq N_{0}$ such that $\mu_{N_{0}^{\prime}} \in$ $U_{1}\left(\mu_{0}\right) \cap U_{2}\left(\mu_{0}\right) \cap U\left(\mu_{0}\right) \cap\left(\mu_{0}+N_{1}\right)$. Noting that (3.9) and (3.10), we obtain

$$
K\left(\mu_{N_{0}^{\prime}}\right) \subseteq K\left(\mu_{0}\right)+U_{1}
$$

and

$$
\left(x^{\prime}+V_{1} \cap W_{1}\right) \cap K\left(\mu_{N_{0}^{\prime}}\right) \neq \emptyset .
$$

By (3.12), we choose

$$
x^{\prime \prime} \in\left(x^{\prime}+V_{1} \cap W_{1}\right) \cap K\left(\mu_{N_{0}^{\prime}}\right) .
$$

Next, we prove that $x^{\prime \prime} \in \widetilde{S}_{\xi}\left(\varepsilon_{N_{0}^{\prime}}, \mu_{N_{0}^{\prime}}\right)$. For any $y^{\prime} \in K\left(\mu_{N_{0}^{\prime}}\right)$, by (3.11), there exists $y_{0} \in K\left(\mu_{0}\right)$ such that $y^{\prime}-y_{0} \in U_{1}$. It follows from (3.13) that $x^{\prime \prime}-x^{\prime} \in V_{1}$. Noting that $\mu_{N_{0}^{\prime}} \in U\left(\mu_{0}\right) \cap$ $\left(\mu_{0}+N_{1}\right)$ and (3.8), we have

$$
F\left(x^{\prime \prime}, y^{\prime}, \mu_{N_{0}^{\prime}}\right) \subseteq \delta_{0} B_{Y}+F\left(x^{\prime}, y_{0}, \mu_{0}\right)
$$

By (3.7), we have

$$
F\left(x^{\prime \prime}, y^{\prime}, \mu_{N_{0}^{\prime}}\right) \subseteq C-\bar{\varepsilon} e+F\left(x^{\prime}, y_{0}, \mu_{0}\right) .
$$

Hence, for any $y \in K\left(\mu_{N_{0}^{\prime}}\right)$ and $z^{\prime \prime} \in F\left(x^{\prime \prime}, y^{\prime}, \mu_{N_{0}^{\prime}}\right)$, there exist $c^{\prime \prime} \in C$ and $z^{\prime} \in F\left(x^{\prime}, y, \mu_{0}\right)$ such that

$$
z^{\prime \prime}=c^{\prime \prime}-\bar{\varepsilon} e+z^{\prime}
$$

It follows from the linearity of $\xi$ that $\xi\left(z^{\prime \prime}\right)+\bar{\varepsilon} \geq \xi\left(z^{\prime}\right)$ for all $\bar{\varepsilon}>0$. This leads to $\xi\left(z^{\prime \prime}\right) \geq \xi\left(z^{\prime}\right)$. Thus

$$
\xi\left(z^{\prime \prime}\right)+\varepsilon_{N_{0}^{\prime}} \geq \xi\left(z^{\prime}\right)+\varepsilon_{N_{0}^{\prime}}=\xi\left(z^{\prime}\right)+\left(\varepsilon_{N_{0}^{\prime}}-\varepsilon_{0}\right)+\varepsilon_{0} \geq 0 .
$$


Hence $x^{\prime \prime} \in \widetilde{S}_{\xi}\left(\varepsilon_{N_{0}^{\prime}}, \mu_{N_{0}^{\prime}}\right)$. Also, since $x^{\prime} \in\left(x_{0}+W_{1}\right)$ and by (3.2) and (3.13), we have

$$
x^{\prime \prime} \in x^{\prime}+V_{1} \cap W_{1} \subseteq x_{0}+W_{1}+W_{1} \subseteq x_{0}+W_{0}
$$

This means that $\left(x_{0}+W_{0}\right) \cap \widetilde{S}_{\xi}\left(\varepsilon_{N_{0}^{\prime}}, \mu_{N_{0}^{\prime}}\right) \neq \emptyset$, which contradicts (3.1). This completes the proof.

Theorem 3.3 We assume that for any given $\xi \in B_{e}^{*}$, there exists $\delta>0$ such that the approximate solution set $\widetilde{S}_{\xi}(\cdot, \cdot)$ exists in $\left[\varepsilon_{0}, \delta\right) \times N\left(\mu_{0}\right)$. Suppose that conditions (i)-(iv) as in Theorem 3.2 are satisfied. Assume further that for each $x \in K\left(\mu_{0}\right), F\left(x, K\left(\mu_{0}\right), \mu_{0}\right)+C$ is a convex set. Then the approximate solution mapping $\widetilde{S}:\left[\varepsilon_{0}, \delta\right) \times N\left(\mu_{0}\right) \rightarrow 2^{X}$ is l.s.c. at $\left(\varepsilon_{0}, \mu_{0}\right)$.

Proof Since $F\left(x, K\left(\mu_{0}\right), \mu_{0}\right)+C$ is a convex set for each $x \in K\left(\mu_{0}\right)$, by virtue of Lemma 3.1, it holds that $\widetilde{S}\left(\varepsilon_{0}, \mu_{0}\right)=\bigcup_{\xi \in B_{e}^{*}} \widetilde{S}_{\xi}\left(\varepsilon_{0}, \mu_{0}\right)$. It follows from Theorem 3.2 that for each $\xi \in$ $B_{e}^{*}, \widetilde{S}_{\xi}(\cdot, \cdot)$ is l.s.c. at $\left(\varepsilon_{0}, \mu_{0}\right)$. Thus, in view of Lemma 2.4 , we obtain that $\widetilde{S}(\cdot, \cdot)$ is l.s.c. at $\left(\varepsilon_{0}, \mu_{0}\right)$.

The following example illustrates all of the assumptions in Theorem 3.3.

Example 3.4 Let $Y=\mathbb{R}^{2}, C=\mathbb{R}_{+}^{2}:=\left\{\left(x_{1}, x_{2}\right) \in \mathbb{R}^{2}: x_{1} \geq 0, x_{2} \geq 0\right\}$ and $Z=X=\mathbb{R}$. Let $B\left(0, \frac{1}{2}\right)$ be the closed ball of radius $1 / 2$ in $\mathbb{R}^{2}$. Let $B=[-2,2], M=[-1,1]$ and the set-valued mapping $F: B \times B \times M \rightarrow 2^{Y}$ be defined by

$$
F(x, y, \mu)=(w(x, y, \mu), v(x, y, \mu))+B(0,1 / 2)
$$

where $w(x, y, \mu):=y^{2}\left(2^{\mu}-1\right)+x(y-x+1)-3 y+2$ and $v(x, y, \mu):=y^{2}\left(2^{\mu}-1\right)-x^{2}+2 x y+3$. Define a set-valued mapping $K: M \rightarrow 2^{X}$ for all $\mu \in M$, by $K(\mu):=[-2+\mu, 2+\mu] \cap[-2,2]$. We choose $e=(1,1) \in \operatorname{int} C, \varepsilon_{0}=2.5, \mu_{0}=0$ and $\xi=(1,0)$. We can see that $B_{(1,1)}^{*}=$ $\left\{\left(x_{1}, x_{2}\right): x_{1}+x_{2}=1, x_{1}, x_{2} \geq 0\right\}$ and $1 \in \widetilde{S}_{(1,0)}\left(\varepsilon_{0}, 0\right)$. Further, for any $\mu \in(-1,1)$, there exists $\varepsilon \in[2.5,4.5)$ such that $1 \in \widetilde{S}_{(1,0)}(\varepsilon, \mu)$. Hence, $\widetilde{S}_{(1,0)}(\cdot, \cdot)$ exists in $[2.5,4.5) \times[-1,1]$. It is easy to observe that for any $y \in K(0), F(\cdot, y, 0)$ is $C$-concave on $K(0)$. Clearly, condition (ii) is true. It is obvious that $K(M)=[-2,2]$. Let $N\left(\mu_{0}\right)=[-1,1]$, we can see that $F(\cdot, \cdot, \cdot)$ is uniformly continuous on $K(M) \times K(M) \times N\left(\mu_{0}\right)$. Finally, we can check that for each $x \in[-2,2], F(x,[-2,2], 0)+C$ is a convex set. Applying Theorem 3.3 , we obtain that $\widetilde{S}$ is l.s.c. at $(2.5,0)$.

The following example illustrates that the concavity of $F$ cannot be dropped.

Example 3.5 Let $Y=\mathbb{R}^{2}, C=\mathbb{R}_{+}^{2}$ and $Z=X=\mathbb{R}$. Let $B=[-2,2], M=[-1,1]$ and the setvalued mapping $F: B \times B \times M \rightarrow 2^{Y}$ be defined by

$$
F(x, y, \mu)=[\mu x(x-y)-0.5,2] \times\{x(x-y)-0.5\}
$$

Define a set-valued mapping $K: M \rightarrow 2^{X}$ for all $\mu \in M$, by $K(\mu):=[0,1]$. We choose $e=(1,1) \in \operatorname{int} C, \varepsilon_{0}=0.5, \mu_{0}=0$. Then, all the assumptions of Theorem 3.3 are satisfied 
except (iii). Indeed, taking $y=1, x_{1}=0, x_{2}=1$ and $t=0.5$, we have

$$
\begin{aligned}
(-2.5,-0.25)= & (-0.5,-0.75)-0.5(2,-0.5)-0.5(2,-0.5) \\
\in & {[-0.5,2] \times\{-0.75\}-0.5([-0.5,2] \times\{-0.5\}) } \\
& -0.5([-0.5,2] \times\{-0.5\}) \\
\in & F(0.5(0)+0.5(1), 1,0)-0.5 F(0,1,0)-0.5 F(1,1,0) \\
= & F(0.5,1,0)-0.5 F(0,1,0)-0.5 F(1,1,0),
\end{aligned}
$$

but $(-2.5,-0.25) \notin C$. The direct computation shows that

$$
\tilde{S}\left(\varepsilon_{0}, \mu\right)= \begin{cases}\{0,1\} & \text { if } \mu \in(0,1], \\ {[0,1]} & \text { if } \mu=0, \\ \{0\} & \text { if } \mu \in[-1,0) .\end{cases}
$$

Clearly, we see that $\tilde{S}(\cdot, \cdot)$ is even not l.s.c. at $\left(\varepsilon_{0}, \mu_{0}\right)$ since $F\left(\cdot, y, \mu_{0}\right)$ is not $C$-concave on $K\left(\mu_{0}\right)$

\section{Competing interests}

The authors declare that they have no competing interests.

\section{Authors' contributions}

All authors read and approved the final manuscript.

\section{Author details}

'Department of Mathematics, Faculty of Science, Naresuan University, Phitsanulok, 65000, Thailand. ${ }^{2}$ Program in Mathematics, Faculty of Education, Pibulsongkram Rajabhat University, Phitsanulok, 65000, Thailand.

\section{Acknowledgements}

The authors were partially supported by the Thailand Research Fund, Grant No. PHD/0078/2554 and Grant No. RSA5780003. The authors would like to thank the referees for their remarks and suggestions, which helped to prove the paper.

\section{Received: 3 July 2014 Accepted: 7 October 2014 Published: 21 Oct 2014}

\section{References}

1. Ansari, QH, Oettli, W, Schläger, D: A generalization of vectorial equilibria. Math. Methods Oper. Res. 46, 147-152 (1997)

2. Ansari, QH, Konnov, IV, Yao, JC: Existence of a solution and variational principles for vector equilibrium problems. J. Optim. Theory Appl. 110(3), 481-492 (2001)

3. Ansari, QH, Yang, XQ, Yao, JC: Existence and duality of implicit vector variational problems. Numer. Funct. Anal. Optim. 22(7-8), 815-829 (2001)

4. Ansari, QH, Konnov, IV, Yao, JC: Characterizations of solutions for vector equilibrium problems. J. Optim. Theory Appl. 113(3), 435-447 (2002)

5. Anh, LQ, Khanh, PQ: Semicontinuity of the solution set of parametric multivalued vector quasiequilibrium problems. J. Math. Anal. Appl. 294, 699-711 (2004)

6. Anh, LQ, Khanh, PQ: Semicontinuity of solution sets to parametric quasivariational inclusions with applications to traffic networks II: lower semicontinuities applications. Set-Valued Anal. 16, 943-960 (2008)

7. Anh, LQ, Khanh, PQ: Sensitivity analysis for weak and strong vector quasiequlibrium problems. Vietnam J. Math. 37, 237-253 (2009)

8. Anh, LQ, Khanh, PQ: Continuity of solution maps of parametric quasiequilibrium problems. J. Glob. Optim. 46 247-259 (2010)

9. Huang, NJ, Li, J, Thompson, HB: Stability for parametric implicit vector equilibrium problems. Math. Comput. Model. 43, 1267-1274 (2006)

10. Cheng, YH, Zhu, DL: Global stability results for the weak vector variational inequality. J. Glob. Optim. 32, 543-550 (2005)

11. Gong, XH, Yao, JC: Lower semicontinuity of the set of efficient solutions for generalized systems. J. Optim. Theory Appl. 138, 197-205 (2008)

12. Chen, $\mathrm{CR}$, Li, SJ, Teo, KL: Solution semicontinuity of parametric generalized vector equilibrium problems. J. Glob. Optim. 45, 309-318 (2009) 
13. Gong, $\mathrm{XH}$ : Continuity of the solution set to parametric weak vector equilibrium problems. J. Optim. Theory Appl. 139 35-46 (2008)

14. Li, SJ, Fang, ZM: Lower semicontinuity of the solution mappings to a parametric generalized Ky Fan inequality. J. Optim. Theory Appl. 147, 507-515 (2010)

15. Gong, XH, Kimura, K, Yao, JC: Sensitivity analysis of strong vector equilibrium problems. J. Nonlinear Convex Anal. 9 83-94 (2008)

16. Li, SJ, Liu, HM, Zhang, Y, Fang, ZM: Continuity of the solution mappings to parametric generalized strong vector equilibrium problems. J. Glob. Optim. 55, 597-610 (2013)

17. Han, Y, Gong, XH: Lower semicontinuity of solution mapping to parametric generalized strong vector equilibrium problems. Appl. Math. Lett. 28, 38-41 (2014)

18. Anh, LQ, Khanh, PQ: Semicontinuity of the approximate solution sets of multivalued quasiequilibrium problems. Numer. Funct. Anal. Optim. 29, 24-42 (2008)

19. Li, XB, Li, SJ: Continuity of approximate solution mappings for parametric equilibrium problems. J. Glob. Optim. 51, $541-548(2011)$

20. Aubin, JP, Ekeland, I: Applied Nonlinear Analysis. Wiley, New York (1984)

21. Berge, C: Topological Spaces. Oliver \& Boyd, London (1963)

22. Chen, $\mathrm{CR}$, Li, SJ, Teo, KL: Solution semicontinuity of parametric generalized vector equilibrium problems. J. Glob. Optim. 45, 309-318 (2009)

23. Jahn, J: Vector Optimization: Theory, Applications and Extensions. Springer, Berlin (2004)

10.1186/1029-242X-2014-421

Cite this article as: Wangkeeree et al.: Lower semicontinuity of approximate solution mappings for parametric generalized vector equilibrium problems. Journal of Inequalities and Applications 2014, 2014:421

\section{Submit your manuscript to a SpringerOpen ${ }^{\circ}$ journal and benefit from:}

- Convenient online submission

- Rigorous peer review

- Immediate publication on acceptance

- Open access: articles freely available online

- High visibility within the field

- Retaining the copyright to your article 